\title{
Study of Mechanical Properties of Composite Material Based on Polypropylene and Vietnamese Rice Husk Filler
}

\author{
Thanh Duy Tran, ${ }^{1}$ Mao Dang Nguyen, ${ }^{1}$ C. N. Ha Thuc, ${ }^{1}$ H. Ha Thuc, ${ }^{2}$ and Tai Dang Tan ${ }^{3}$ \\ ${ }^{1}$ Faculty of Material Science, University of Science, Vietnam National University (VNU-HCM), Ho Chi Minh City, \\ 227 Nguyen Van Cu Road, Ho Chi Minh City, Vietnam \\ ${ }^{2}$ Faculty of Chemistry, University of Science, Vietnam National University (VNU-HCM), Ho Chi Minh City, \\ 227 Nguyen Van Cu Road, Ho Chi Minh City, Vietnam \\ ${ }^{3}$ Plastic and Rubber Technology Center, 156 Nam Ky Khoi Nghia Road, Ho Chi Minh City, Vietnam \\ Correspondence should be addressed to C. N. Ha Thuc; htcnhan@hcmus.edu.vn
}

Received 28 December 2012; Revised 22 May 2013; Accepted 10 June 2013

Academic Editor: Davide Vione

Copyright (C) 2013 Thanh Duy Tran et al. This is an open access article distributed under the Creative Commons Attribution License, which permits unrestricted use, distribution, and reproduction in any medium, provided the original work is properly cited.

\begin{abstract}
Nowadays, the consideration of the environmental impact of polymer products has led to the development of new materials combining polymers and renewable resources. Among these materials, biocomposites have been extensively studied. In the current work, we focused on preparation of biocomposites based on polypropylene and Vietnamese rice husk flour having narrow size distribution $(100-125 \mu \mathrm{m})$ with the presence of maleic anhydride grafted polypropylene as a compatibilizer. The effect of rice husk flour and maleic anhydride grafted polypropylene content on properties of composites was investigated. The results showed that mechanical parameters as well as thermal stability were significantly improved at relatively high rice husk content by the addition of maleic anhydride grafted polypropylene.
\end{abstract}

\section{Introduction}

Nowadays, natural resources have various applications in eco-friendly composite materials because they have many advantages, such as low cost, low energy consumption, low density, high-specific mechanical properties, and nonabrasive and especially biodegradable ability when compared to synthetic fibers $[1,2]$. Therefore, the development of biocomposites has been extensively exploited [3-5]. Rice husk flour (RHF) is one of the main agricultural residues eliminated during rice production. Each year, a large amount of rice straw and rice husk approximating about 685 million tons and 137 million tons, respectively, is eliminated by many rice factories in the world [6], especially in Asia region, leading to environmental problems. There have been various researches to develop applications of RHF as well as minimize environmental pollution caused by RHF. For example, RHF has been used as the energy source in some countries [7]. Besides, RHF has been also applied for obtaining rice husk ash or silica ash, and then these products have been used as the filler in polymer composites, ceramic or road-building materials, and adsorbents of heavy metals $[7,8]$. In recent years, the utilization of RHF in polymer composites has been attracting much attention [9-11], and RHF could be used as the effective biodegradable filler in polymer composites.

The current study exploited the application of Vietnamese RHF source having narrow size distribution $(100-125 \mu \mathrm{m})$ in composites based on polypropylene (PP) matrix with the presence of maleic anhydride grafted polypropylene (PPg-MA) compatibilizer. After PP-g-MA was added to the composites, the anhydride groups of PP-g-MA would react with hydroxyl groups on surface of RHF particles. As a result, PP-g-MA acted as the copolymer to improve matrix/filler interfacial adhesion, thus leading to improvement of composite properties. Various characterization techniques, such as Fourier transform infrared spectroscopy (FTIR), scanning electron microscopy (SEM), thermal gravimetric analysis (TGA), and tensile test, were performed to explore the effect of RHF and PP-g-MA on final properties of the composites. 


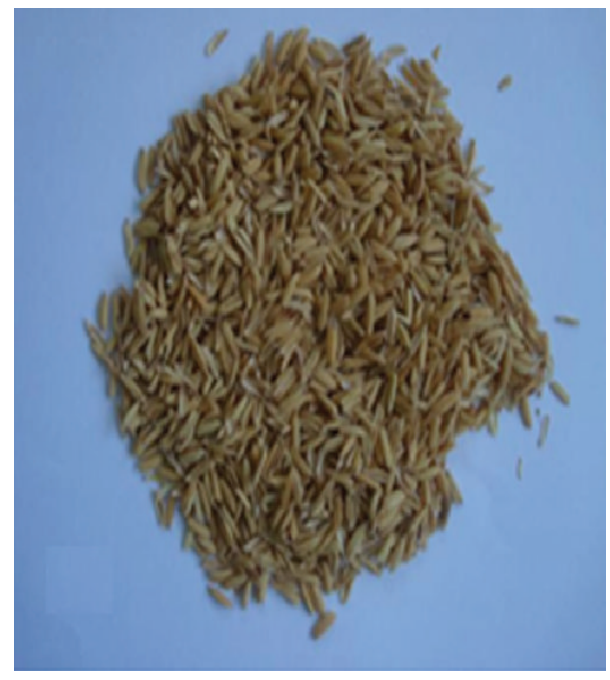

(a)

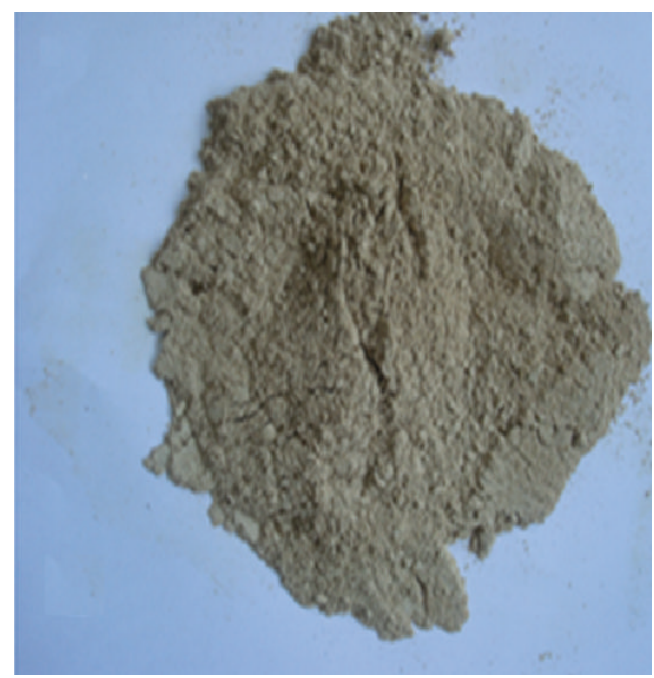

(b)

FIGURE 1: NaOH-treated RHF before (a) and after (b) grinding and screening.

\section{Experimental Methods}

2.1. Materials. Isotactic PP was supplied by Parshwa Polymer Industries company (India) in the form of pellets with a density of $0.92-0.95 \mathrm{~g} / \mathrm{cm}^{3}$, melting temperature $165-170^{\circ} \mathrm{C}$ and a melt flow index $2.3 \mathrm{~g} / 10$ minute. RHF was from Tien Giang rice factory (Vietnam). PP-g-MA with melting temperature $162^{\circ} \mathrm{C}$ and melt flow index $120 \mathrm{~g} / 10$ minute was provided by DuPont Corporation (USA), in the trademark Fusabond P M613-05.

\subsection{Experiment}

2.2.1. RHF Treatment Process by Sodium Hydroxide (NaOH) Solution. RHF was previously washed to remove some dust, soil, and rocks. After that it was treated by $\mathrm{NaOH} 1 \%$ solution for 72 hours. The solution was then neutralized by hydrogen chloride $0.1 \mathrm{M}$ solution, and RHF was taken out and washed 3 times by water. Finally RHF was dried at $80^{\circ} \mathrm{C}$ for 24 hours in a vacuum oven to remove moisture before it was used in the following steps.

2.2.2. Preparation of the Composite Materials PP/RHF. The treated RHF was grinded and screened on two types of sieves having mesh sizes of 100 and $125 \mu \mathrm{m}$ to collect RHF particles which had size distribution in the range 100$125 \mu \mathrm{m}$. All components, such as PP, RHF, and PP-g-MA, were simultaneously blended in a Haake PolyLab mixer at stir rate $60 \mathrm{rpm}$ and temperature $170^{\circ} \mathrm{C}$ for 6 minutes. After that, the melted compound was immediately pressed between two plates of a hot press at $180^{\circ} \mathrm{C}$ and $3 \mathrm{kPa}$ for 5 minutes to prepare composite layer having size $17 \mathrm{~cm}$ length $\times 17 \mathrm{~cm}$ width $\times 2 \mathrm{~mm}$ thickness. Finally, two plates of the press were cooled down to room temperature, and the composite layer was taken out to use for testing properties.

2.2.3. Characterization Techniques. The chemical structure of the untreated and $\mathrm{NaOH}$-treated RHF was analyzed by IR absorption spectroscopy, using a Tensor 27 FTIR spectrometer (Bruker Optics Co., USA) with $\mathrm{KBr}$ disc. The scanning was performed at $2 \mathrm{~cm}^{-1}$ resolution.

The morphology of brittle-fractured surfaces of composites was observed by scanning electron microscope JSM 6600 (JEOL co., Japan).

Tensile and flexural strength were investigated according to ASTM D638 and ASTM D790, respectively, at room temperature by using an apparatus QC-505A1 (Comtech, Taiwan) with $5 \mathrm{~mm} / \mathrm{min}$ crosshead speed. At least 5 pieces were measured for each composition.

Thermal stability of the materials was characterized by using a thermogravimetric analyzer TGA Q500 (TA Instruments co., USA). The samples were under nitrogen atmosphere using the temperature range of $35-800^{\circ} \mathrm{C}$ and heating rate of $10^{\circ} \mathrm{C} / \mathrm{min}$.

\section{Results and Discussion}

3.1. Effect of $\mathrm{NaOH}$ Treatment on Structure of RHF. Figure 1 shows the shape of RHF before and after grinding and screening.

As suggested in the literature, the $\mathrm{NaOH}$ treatment is needed to remove the hemicelluloses, lignin, and other impurities in natural fibers [12] as well as in rice husk [13]. As a result, the RHF surface becomes rough, and hydrogen bonds between hydroxyl groups of the celluloses of RHF may be collapsed; thus hydroxyl groups become active to react with anhydride groups of PP-g-MA.

The result of FT-IR spectrum of the untreated and treated RHF is shown in Figure 2. The specific stretching vibration of the hydroxyl groups of cellulose at $3200-3500 \mathrm{~cm}^{-1}$ is virtually unchanged. However, there is a noticeable decrease in the intensity of peak at $1700-1750 \mathrm{~cm}^{-1}$ which is corresponding to the stretching vibration of $-\mathrm{C}=\mathrm{O}$ bonding of hemicelluloses and lignin. In addition, peak positions at $1400-1600 \mathrm{~cm}^{-1}$, assigned $\mathrm{C}=\mathrm{C}$ stretching of aromatic 


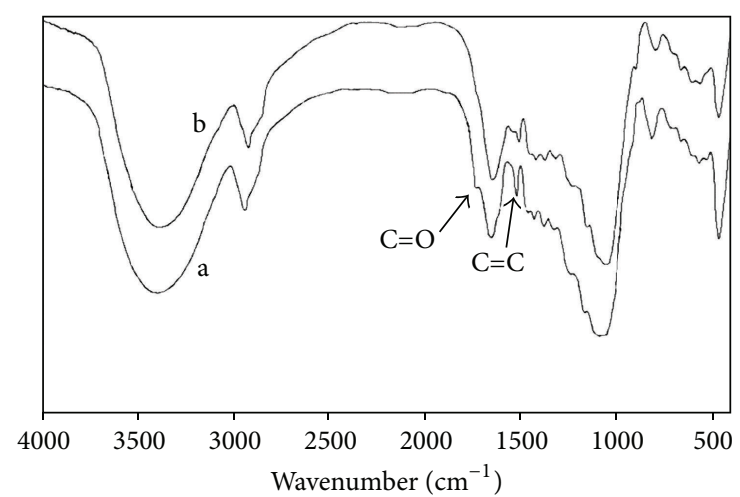

Figure 2: FT-IR spectrum of (a) untreated RHF and (b) $\mathrm{NaOH}-$ treated RHF in 72 hours.

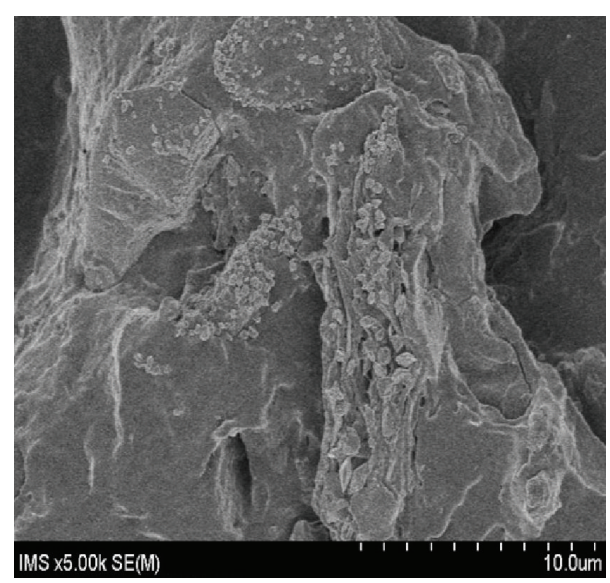

(a)

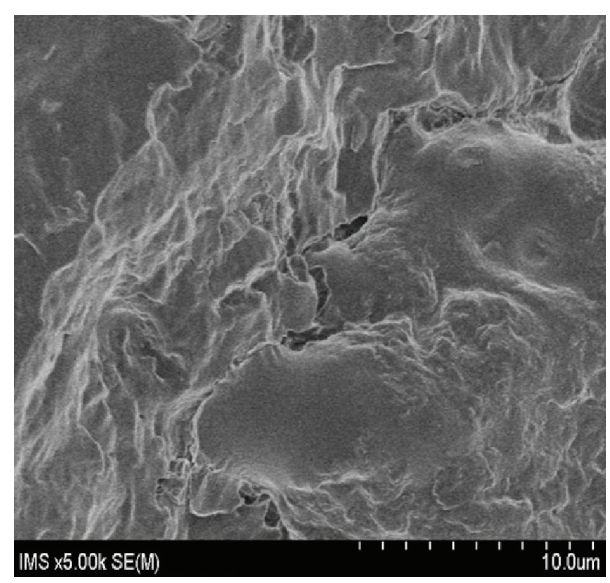

(b)

FIgURE 3: Surface of untreated RHF (a) and $\mathrm{NaOH}$-treated RHF (b).

carbon of lignin, seem to be slightly dropped. From these observations, it is suggested that the amount of $\mathrm{C}=\mathrm{O}$ and $\mathrm{C}=\mathrm{C}$ groups which are from hemicelluloses and lignin components is reduced by the treatment with $\mathrm{NaOH} 1 \%$ solution.

The SEM micrograph in Figure 3 shows the surface of untreated and treated RHF. In case of untreated RHF, the surface indicates the presence of clustered impurities that may be lignin and hemicellulose existing on structure (Figure 3(a)). However, after treatment (Figure 3(b)) those components are removed, thus leaving a clean surface of RHF particles.

\subsection{Mechanical Properties of PP/RHF Composite Materials}

3.2.1. Effect of PP-g-MA Content on the Mechanical Properties of Composites. The effect of PP-g-MA content on the mechanical parameters of the composites is shown in Figures 4 and 5. Generally, the mechanical behavior of the composites is improved with the addition of PP-g-MA [14]. The mechanical behavior of composites has tendency to increase according to the change of PP-g-MA content from 0 to $4 \mathrm{wt} \%$, and then it decreases at 6 and $8 \mathrm{wt} \%$ of PP-g-MA, but it is still higher when compared to the case of composites without compatibilizer. The positive effect at low PP-g-MA content could result from the reaction of the hydrophilic hydroxyl groups of the RHF and the anhydride groups of PP-g-MA through esterification reaction. This phenomenon acts as the effective factor for improving interfacial adhesion between filler and matrix as reported in the literature [15]. Meanwhile, at higher content of PP-g-MA, the excess of the compatibilizer amount may lead to the agglomeration and formation of a new PP-g-MA phase. Consequently, this may inhibit the interfacial adhesion between RHF and PP [16].

The influence of PP-g-MA content on the toughness of the composites is shown in Figure 5. The toughness of composites also has similar tendency to tensile and flexural strength when PP-g-MA content changes.

3.2.2. Effect of RHF Content on Mechanical Properties of PP/ RHF Composites. Figure 6 shows the relationship between the mechanical properties and the RHF content in PP/RHF composites. The RHF content is changed from 0 to $120 \mathrm{phr}$ in the presence of $4 \mathrm{wt} \%$ compatibilizer. The results indicate that the increase in filler content leads to the improvement of composite stiffness. This is a normal behavior when rigid fillers are incorporated into polymer matrix in case of microcomposite [17] as well as nanocomposites [18]. The stress of composites is better when compared to virgin PP (at filler content $0 \mathrm{phr}$ ). Stress increases when filler content is up to $100 \mathrm{phr}$ and then diminishes at higher amount of filler. These behaviors are due to the fact that RHF well disperses into polymer matrix at content from 40 to $100 \mathrm{phr}$ under chemical linking effect of PP-g-MA, and then RHF acts as the nucleus to absorb energy caused by external load. But at higher RHF content, such as $120 \mathrm{phr}$, the agglomeration of filler appears in the matrix, thus leading to the reduction of interfacial adhesion between filler and matrix. In this case, the content of PP-g-MA at $4 \mathrm{wt} \%$ is not sufficient to well support the dispersion of filler into matrix. As a result, this phenomenon causes decrease in stress of materials. In this case, the toughness in Figure 7 also shows a similar trend to tensile and flexural strength of composites.

To have better observation about the effect of PP-g-MA on structure and properties of composites, the morphology of brittle-fractured surfaces of PP/RHF (100/100) composites 

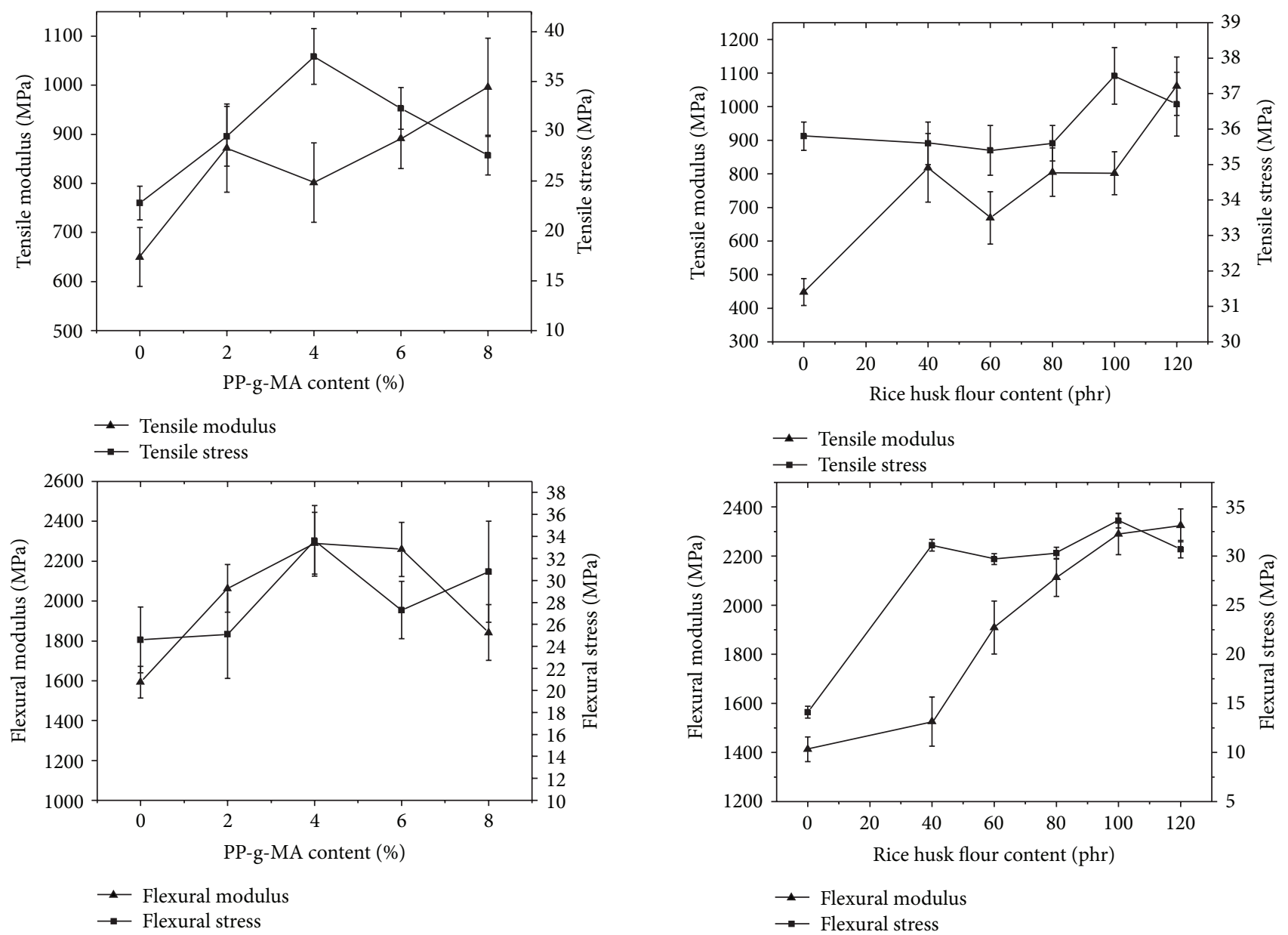

FIGURE 4: Effect of PP-g-MA content on tensile and flexural strength of PP/RHF (100/100) composites.

FIGURE 6: Effect of RHF content on tensile and flexural strength of $\mathrm{PP} / \mathrm{RHF}$ composites in the presence of $4 \mathrm{wt} \% \mathrm{PP}-\mathrm{g}$-MA.

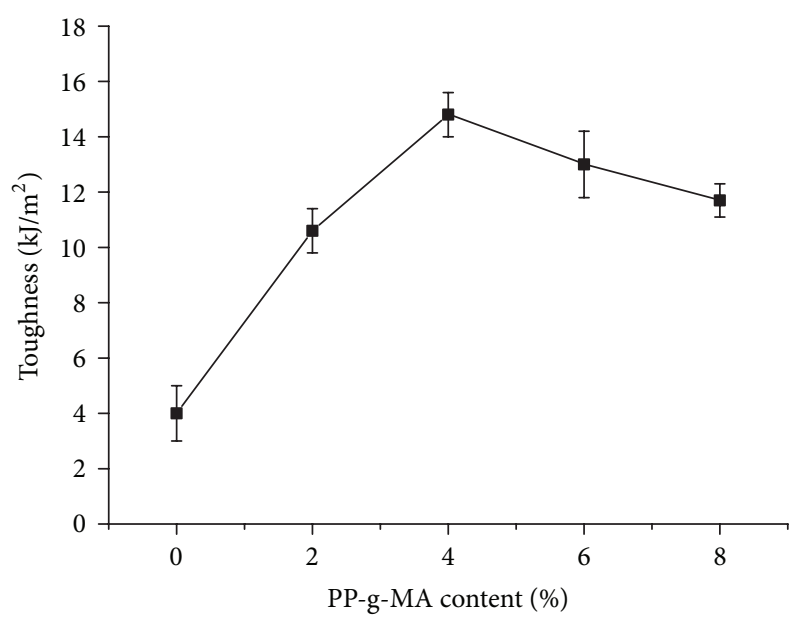

FIGURE 5: Effect of PP-g-MA content on toughness of PP/RHF $(100 / 100)$ composites.

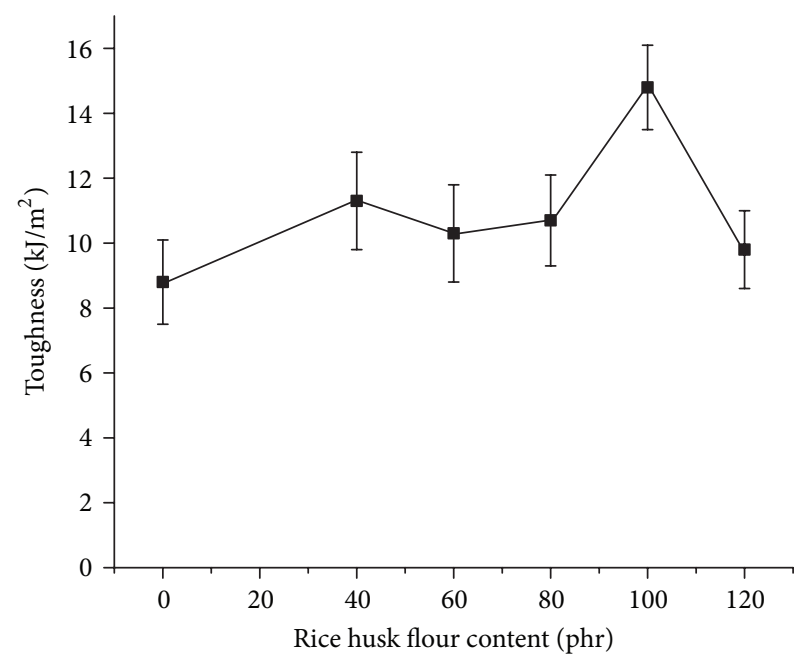

FIGURE 7: Effect of RHF content on toughness of PP/RHF composites in the presence of $4 \mathrm{wt} \%$ PP-g-MA. 


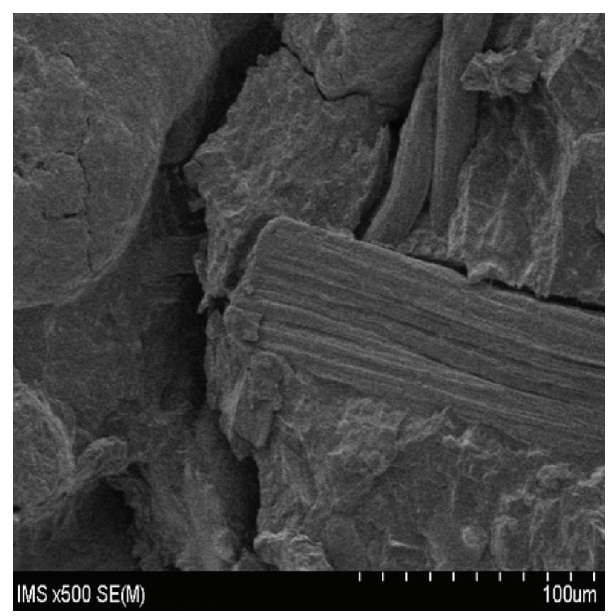

(a)

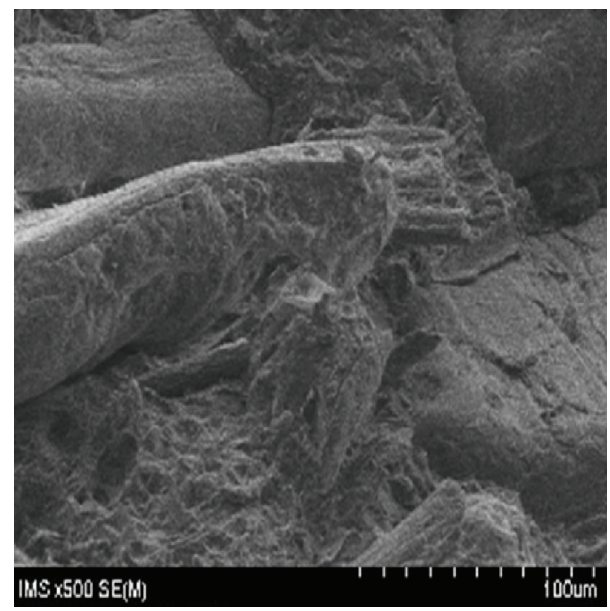

(b)

Figure 8: SEM images of (a) PP/RHF and (b) PP/RHF/PP-g-MA $4 \mathrm{wt} \%$.

is also studied by SEM and shown in Figure 8. In the absence of PP-g-MA, the composites display a rough morphology with appearance of the phase separation between the RHF particles and PP matrix (Figure 8(a)). This clearly shows the poor interaction between filler and matrix phase. In the presence of $4 \mathrm{wt} \%$ PP-g-MA, the morphological observation demonstrates that the compatibilizer reduces the separating positions and there are no free spaces between filler and the polymer matrix. This finding shows that PP-g-MA is a promising factor to improve adhesion at the interfacial region between filler and matrix.

3.3. Thermal Stability of PP/RHF (100/100) Composites. From the curve (a) (Figure 9), we can see that the mass loss of $\mathrm{PP}$ occurs at $384^{\circ} \mathrm{C}$ and gains maximum value at $454^{\circ} \mathrm{C}$. In curve (b), there is a slight change of RHF weight starting from $100^{\circ} \mathrm{C}$ to under $200^{\circ} \mathrm{C}$, corresponding to the evaporation of absorbed moisture existing in RHF. The real mass loss due to

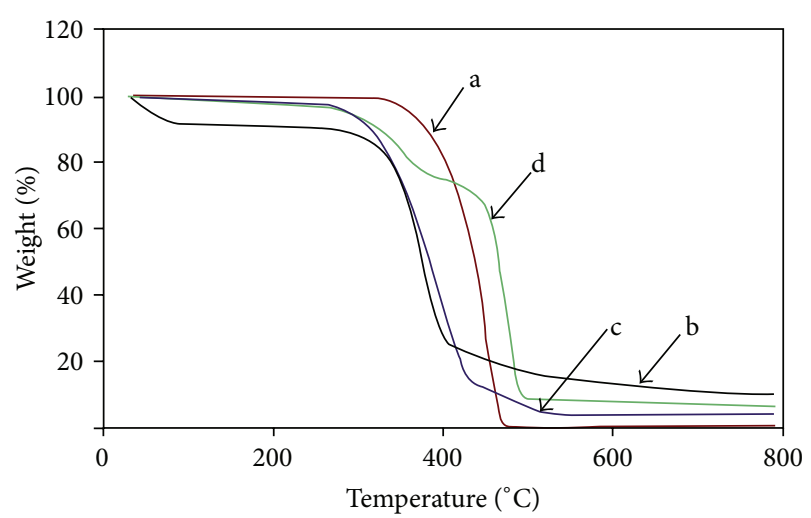

Figure 9: Thermograms of (a) PP, (b) RHF, (c) PP/RHF, and (d) PP/RHF/PP-g-MA.

the decomposition of the cellulose/hemicelluloses and lignin in RHF only begins with two temperature steps at 250 and $344^{\circ} \mathrm{C}$, respectively. As seen in curves (c) and (d), the mass loss of the PP/RHF/PP-g-MA composites begins at $295^{\circ} \mathrm{C}$, higher than that of PP/RHF composites, the mass loss of which begins at $285^{\circ} \mathrm{C}$. In addition, the mass loss rate of $\mathrm{PP} / \mathrm{RHF} / \mathrm{PP}-\mathrm{g}-\mathrm{MA}$ is also slower than that of PP/RHF. These results indicate that PP/RHF/PP-g-MA has better thermal stability in comparison with PP/RHF. The reason may be that the addition of $4 \mathrm{wt} \%$ PP-g-MA helps RHF disperse more easily into PP matrix through the improvement of interfacial adhesion by esterification reaction between hydroxyl groups of RHF and the anhydride functional group of PP-g-MA. This phenomenon causes RHF to be well surrounded by PP molecules, and that reduces contact between RHF and heat source, thus improving the thermal resistance of PP/RHF/PPg-MA. These results were also consistent with data reported by Zabihzadeh et al. [15].

\section{Conclusion}

This study shows that the addition of PP-g-MA to composites based on $\mathrm{NaOH}$-treated RHF filler having size range 100$125 \mu \mathrm{m}$ and PP matrix does not only improve tensile and flexural strength but also improve toughness of composites. Besides, the thermal stability of composite is also enhanced in the presence of compatibilizer. These results show that PP-gMA acts as the effective factor to improve interfacial adhesion between PP and RHF filler. In Vietnam, RHF is extremely widespread and available for many applications. The results of this research show that Vietnamese RHF is also a promising renewable resource to be applied for polymer composites.

\section{Acknowledgments}

The study was supported by the Department of Material Sciences, Ho Chi Minh University of Science. The authors thank all participants for their enthusiasm and cooperation in the study. 


\section{References}

[1] H. P. S. Abdul Khalil, I. U. H. Bhat, M. Jawaid, A. Zaidon, D. Hermawan, and Y. S. Hadi, "Bamboo fibre reinforced biocomposites: a review," Materials and Design, vol. 42, pp. 353368, 2012.

[2] T. Huber, J. Müssig, O. Curnow, S. Pang, S. Bickerton, and M. P. Staiger, "A critical review of all-cellulose composites," Journal of Materials Science, vol. 47, no. 3, pp. 1171-1186, 2012.

[3] D. T. Tran, D. M. Nguyen, C. N. H. Thuc, and T. T. Dang, "Effect of coupling agents on the properties of bamboo fiber reinforced unsaturated polyester resin composites," Composite Interfaces, vol. 20, no. 5, pp. 343-353, 2013.

[4] Y. Xie, C. A. S. Hill, Z. Xiao, H. Militz, and C. Mai, "Silane coupling agents used for natural fiber/polymer composites: a review," Composites A, vol. 41, no. 7, pp. 806-819, 2010.

[5] P. J. Herrera-Franco and A. Valadez-González, "Mechanical properties of continuous natural fibre-reinforced polymer composites," Composites A, vol. 35, no. 3, pp. 339-345, 2004.

[6] J. S. Lim, Z. Abdul Manan, S. R. Wan Alwi, and H. Hashim, "A review on utilisation of biomass from rice industry as a source of renewable energy," Renewable and Sustainable Energy Reviews, vol. 16, no. 5, pp. 3084-3094, 2012.

[7] N. Kayal, P. K. Sinha, and D. Kundu, "Application of chemically modified rice husk for the removal of heavy metals from aqueous solution," Journal of Environmental Science and Engineering, vol. 52, no. 1, pp. 15-18, 2010.

[8] V. H. Le, C. N. H. Thuc, and H. H. Thuc, "Synthesis of silica nanoparticles from Vietnamese rice husk by sol-gel method," Nanoscale Research Letters, vol. 8, article 58, 2013.

[9] H.-S. Yang, H.-J. Kim, J. Son, H.-J. Park, B.-J. Lee, and T.S. Hwang, "Rice-husk flour filled polypropylene composites; mechanical and morphological study," Composite Structures, vol. 63, no. 3-4, pp. 305-312, 2004.

[10] S. M. L. Rosa, S. M. B. Nachtigall, and C. A. Ferreira, "Thermal and dynamic-mechanical characterization of rice-husk filled polypropylene composites," Macromolecular Research, vol. 17, no. 1, pp. 8-13, 2009.

[11] J. Prachayawarakorn and N. Yaembunyng, "Effect of recycling on properties of rice husk-filled-polypropylene," Journal of Science and Technology, vol. 27, pp. 343-352, 2005.

[12] L. Börås and P. Gatenholm, "Surface composition and morphology of CTMP fibers," Holzforschung, vol. 53, no. 2, pp. 188-194, 1999.

[13] E. Guilbert-García, R. Salgado-Delgado, N. A. Rangel-Vázquez, E. García-Hernández, E. Rubio-Rosas, and R. SalgadoRodríguez, "Modification of rice husk to improve the interface in isotactic polypropylene composites," Latin American Applied Research, vol. 42, pp. 83-87, 2012.

[14] Z. Xiuju, S. Juncai, Y. Huajun, L. Zhidan, and T. Shaozao, "Polypropylene hybrid composites filled by wood flour and short glass fiber: effect of compatibilizer on structure and properties," Journal of Thermoplastic Composite Materials, vol. 26, pp. 16-29, 2013.

[15] S. M. Zabihzadeh, G. Ebrahimi, and A. A. Enayati, "Effect of compatibilizer on mechanical, morphological, and thermal properties of chemimechanical pulp-reinforced PP composites," Journal of Thermoplastic Composite Materials, vol. 24, no. 2, pp. 221-231, 2011.

[16] H.-S. Kim, B.-H. Lee, S.-W. Choi, S. Kim, and H.-J. Kim, "The effect of types of maleic anhydride-grafted polypropylene
(MAPP) on the interfacial adhesion properties of bio-flourfilled polypropylene composites," Composites A, vol. 38 , no. 6 , pp. 1473-1482, 2007.

[17] M. Bhowmick, S. Mukhopadhyay, and R. Alagirusamy, "Mechanical properties of natural fibre-reinforced composites," Textile Progress, vol. 44, pp. 85-140, 2012.

[18] T. D. Thanh, N. D. Mao, N. T. K. Ngan, H. T. C. Nhan, H. T. Huy, and A. C. Grillet, "Study structure and properties of nanocomposite material based on unsaturated polyester with clay modified by poly (ethylene oxide)," Journal of Nanomaterials, vol. 2012, Article ID 841813, 5 pages, 2012. 

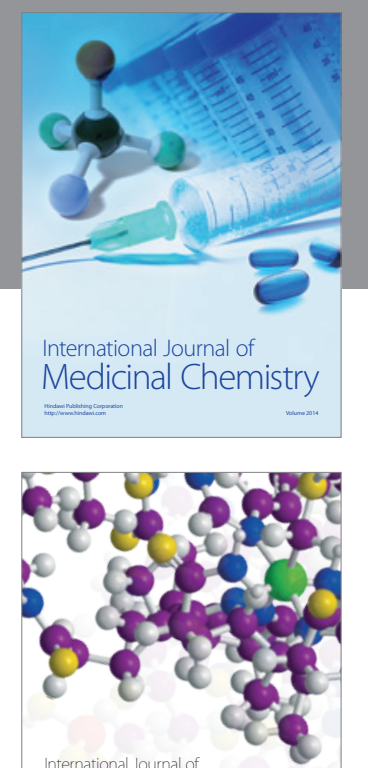

\section{Carbohydrate} Chemistry

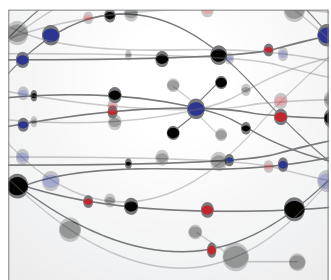

The Scientific World Journal
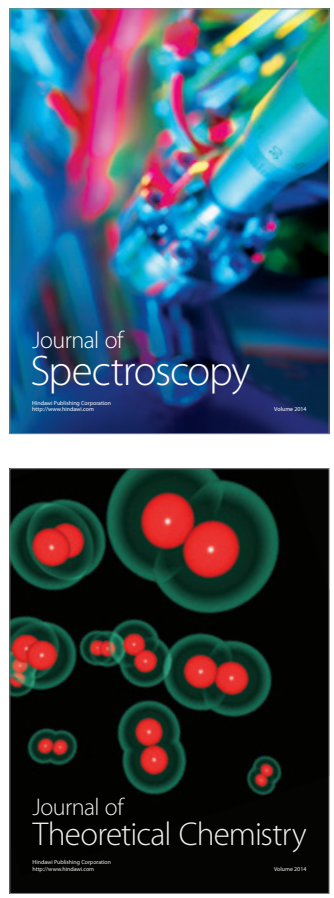
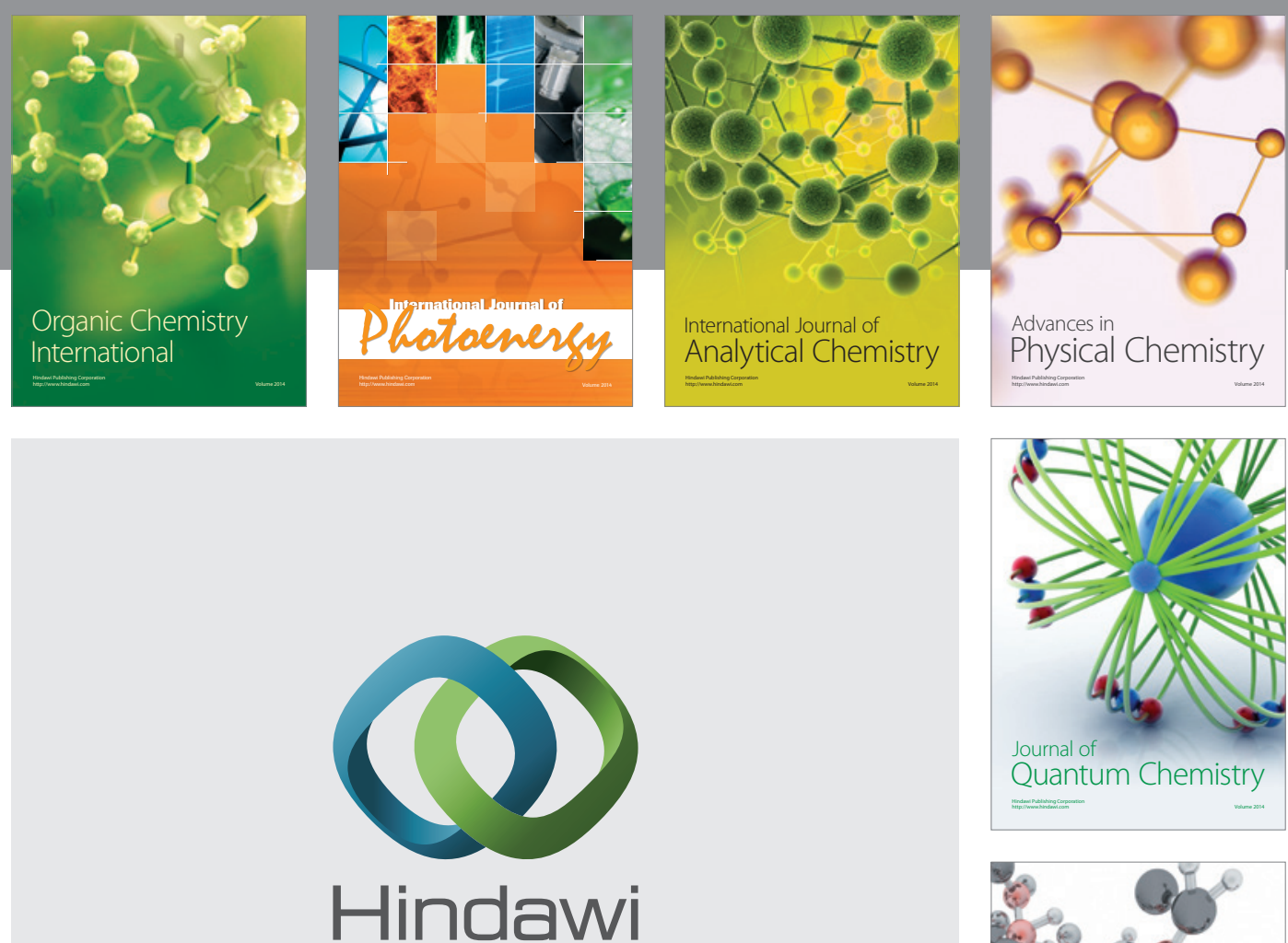

Submit your manuscripts at

http://www.hindawi.com

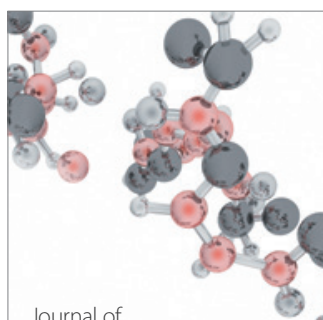

Analytical Methods

in Chemistry

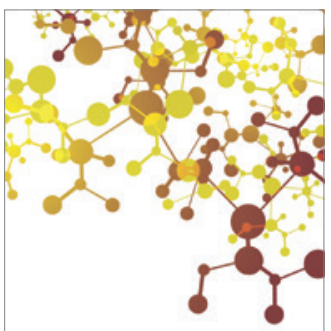

Journal of

Applied Chemistry

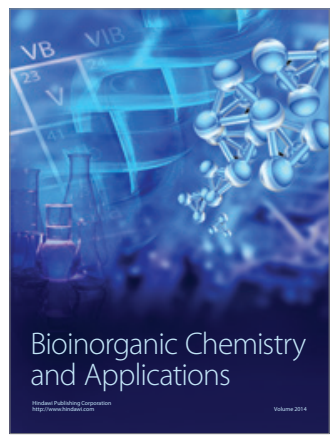

Inorganic Chemistry
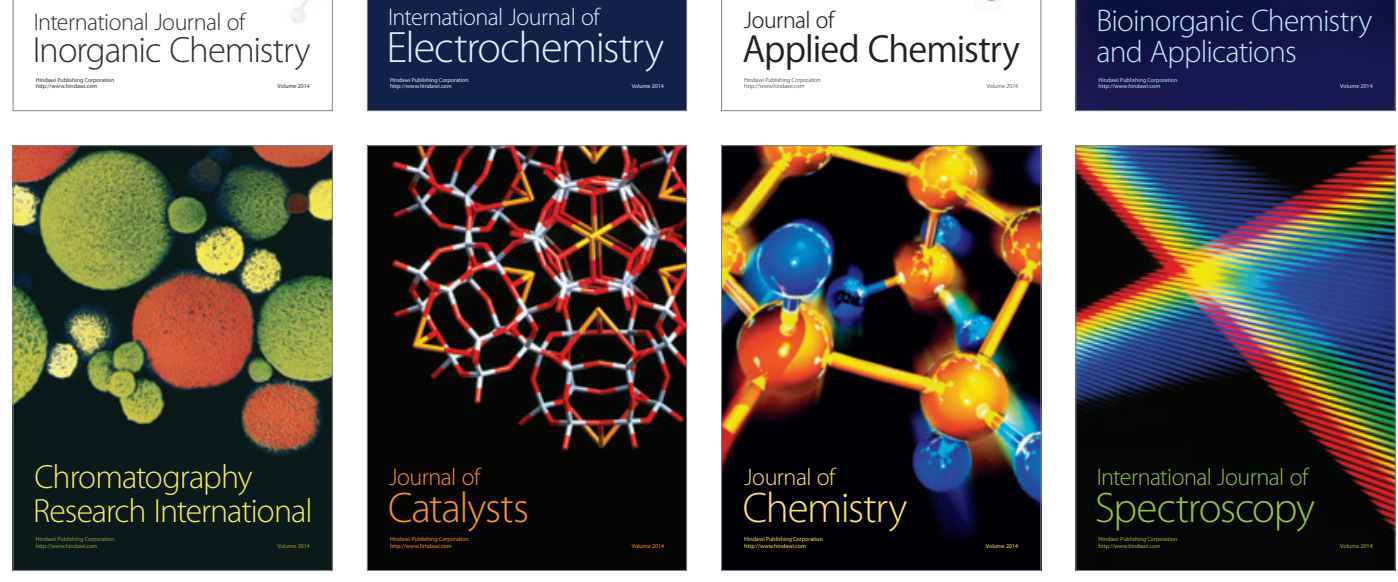